\title{
PREDIÇÃO DE PARÂMETROS E VALORES GENÉTICOS PARA CARACTERES DE CRESCIMENTO E PRODUÇÃO DE LÁTEX EM PROGÊNIES DE SERINGUEIRA $\left({ }^{1}\right)$
}

\author{
REGINALDO BRITO DA COSTA $\left({ }^{2 *}\right)$; MARCOS DEON VILELA DE RESENDE $\left({ }^{3}\right)$; \\ PAULO DE SOUZA GONÇALVES $\left({ }^{4}\right)$; RAUL ALFFONSO RODRIGUES ROA $\left({ }^{5}\right)$; \\ KÊNIA CRISTINE DE OLIVEIRA FEITOSA $\left({ }^{6}\right)$
}

\begin{abstract}
RESUMO
O trabalho objetivou estimar parâmetros e valores genéticos para os caracteres altura, diâmetro, produção de látex e produtividade de progênies de seringueira. As progênies meio-irmãos foram estabelecidas sob delineamento de blocos ao acaso, com 28 tratamentos (progênies), cinco repetições e dez plantas por parcela. Aos três anos de idade, as progênies foram avaliadas quanto aos caracteres: a) altura total $(\mathrm{cm})$; b) diâmetro $(\mathrm{mm})$; c) Produção de borracha seca $(\mathrm{g})$ e produtividade $\left(\mathrm{g} \mathrm{cm}^{-2}\right)$. As estimativas dos coeficientes de herdabilidades individuais $(0,33 ; 0,24$ e 0,51$)$ para os caracteres altura, diâmetro e produtividade, respectivamente, foram consideradas expressivas. Para o caráter produção de borracha seca, a estimativa do coeficiente de herdabilidade individual, no sentido restrito (9\%), embora de baixa magnitude, revela excelente possibilidade de seleção, pois conduziram a estimativa da herdabilidade, em nível de médias de família, a um valor equivalente a $68 \%$. A acurácia entre os valores genéticos preditos e os verdadeiros foram de 0,80 para altura, 0,76 para diâmetro, 0,62 para produção de borracha seca e 0,86 para produtividade. As estimativas de herdabilidades individuais associadas às de médias de progênies podem maximizar os ganhos genéticos com a seleção na população. A inclusão do caráter produtividade foi promissora e deve ser utilizada na seqüência das avaliações, subsidiando o programa de melhoramento genético da espécie no Estado de Mato Grosso do Sul.
\end{abstract}

Palavras-chave: Hevea brasiliensis, parâmetros genéticos, herdabilidade, ganho genético.

(1) Recebido para publicação em 16 de janeiro de 2009 e aceito em 13 de outubro de 2009.

$\left(^{2}\right)$ Universidade Federal de Mato Grosso (UFMT), Faculdade de Engenharia Florestal. Programa de Mestrado em Ciências Florestais e Ambiental. Av. Fernando Corrêa, s/n, 78060-900 Coxipó (MT). E-mail: reg.brito.costa@gmail.com (*) Autor correspondente;

$\left({ }^{3}\right)$ Embrapa - Centro Nacional de Pesquisa de Florestas (CNPF), Caixa Postal 31, 83411-000 Colombo (PR). E-mail: marcos.deon@gmail.com.

$\left({ }^{4}\right)$ Programa Seringueira - Instituto Agronômico de Campinas (IAC), Caixa Postal 28, 13001-970 Campinas (SP). E-mail: paulog@iac.sp.gov.br.

${ }^{5}$ ) Universidade Estadual Paulista Júlio de Mesquita Filho (UNESP), Programa de Pós-Graduação em Agronomia, Av. Brasil, 56, 15385-000 Ilha Solteira (SP). E-mail: roabio@gmail.com.

$\left(^{6}\right)$ UNIVAG - Centro Universitário. Av. Dom Orlando Chaves, n. ${ }^{\circ}$ 2.655, 78118-900 Várzea Grande (MT). E-mail: feitosa.kenia@gmail.com. 


\title{
ABSTRACT \\ GENETIC PARAMETERS AND VALUES PREDICTION FOR GROWTH AND LATEX PRODUCTION TRAITS IN RUBBER TREE PROGENIES
}

\begin{abstract}
This study aimed to estimate genetic values and parameters for plant height, diameter, rubber yield and productivity $\left(\mathrm{g} \mathrm{cm}^{-2}\right)$ of rubber tree progenies. Half-sib progenies were established in a complete randomized block, with 28 treatments, five replicates and 10 plants per plot. At three years of life, the progenies were evaluated regarding the following characters: diameter, rubber production and productivity $\left(\mathrm{g} \mathrm{cm}^{2}\right)$. The estimative coefficients of individual heritabilities $(0.33 ; 0.24$ and 0.51$)$ for plant height, diameter and area production, respectively, were considered expressive. For the character of rubber yield the estimative of the coefficient of individual heritability, towards restrictive (9\%), although of low magnitude, indicates an excellent chance once selection, it led to an estimate of heritability at family average level, to an equivalent value of $68 \%$. The accuracy among the genetics values predicted and the true ones were of 0.80 for height, 0.76 for diameter, 0.62 for rubber yield and 0.86 for productivity ( $g$ $\mathrm{cm}^{2}$ ). The estimative of individual heritability associated with the average of progenies can maximize the genetic gains with selection in to the population. The inclusion of productivity character is promising and should be considered in future evaluations, supporting the species genetic improvement program in Mato Grosso do Sul State.
\end{abstract}

Key words: Hevea brasiliensis, genetic parameters, heritability, genetic gain.

\section{INTRODUÇÃO}

No Brasil, a seringueira [Hevea brasiliensis (Willd ex Adr. de Juss.) Muell.-Arg.], tem como principais áreas de cultivo, as denominadas áreas de escape ao mal-das-folhas (Microcyclus ulei) e localizam-se nos seguintes Estados: São Paulo, Mato Grosso, Mato Grosso do Sul, Bahia e no noroeste do Paraná (Pereira, 1992).

Apesar da importância econômica do cultivo da seringueira para o Estado do Mato Grosso do Sul, ainda há carência de informações que disponibilizem material genético melhorado para pequenos e médios produtores rurais do Estado. Desse modo, o programa de melhoramento da espécie deverá atender, em sua sequência, a demanda crescente de germoplasmas superiores na região.

Os testes de progênies têm sido usados na estimação de parâmetros genéticos e seleção de indivíduos, quando se procura avaliar a magnitude e a natureza da variância genética disponível com vistas a quantificar e maximizar os ganhos genéticos, utilizando-se procedimento de seleção adequado.

A acurada predição de valores individuais de candidatos para seleção é parte essencial em programas de melhoramento genético florestal (RESENDE, 2002); A metodologia Reml/Blup, desenvolvida para o melhoramento de plantas perenes tem maximizado os ganhos genéticos com seleção (RESENDE, 2002, COSTA et al., 2000a; CostA et al., 2005; Missio et al., 2005), por se tratar de um procedimento estimativo, especialmente, para dados desbalanceados, predizendo valores genéticos dos indivíduos em testes de progênies (Resende e FernANDEs, 1999).
Este estudo objetivou estimar parâmetros e valores genéticos para os caracteres altura, diâmetro, produção de látex e produtividade de progênies de seringueira (Hevea brasiliensis). Tais estimativas visam subsidiar o programa de melhoramento genético da espécie no Estado de Mato Grosso do Sul.

\section{MATERIAL E MÉTODOS}

O teste de progênies foi instalado no município de Dois Irmãos do Buriti (MS), localizado na latitude $20^{\circ} 29^{\prime} 14^{\prime \prime} \mathrm{S}$, longitude $55^{\circ} 30^{\prime} 40^{\prime \prime} \mathrm{W}$ e altitude de $320 \mathrm{~m}$. A temperatura média anual é de $27^{\circ} \mathrm{C}$ e a pluviosidade média anual é de $1370 \mathrm{~mm}$. O tipo de solo do local do experimento é o podzólico vermelho-amarelo com textura arenosa/média e baixa fertilidade natural. Os fertilizantes usados foram uréia, superfosfato triplo e cloreto de potássio conforme recomendação de RAIJ et al. (1997). Em relação aos tratos culturais, aplicaram-se herbicidas específicos para o controle das plantas daninhas, desbrota para livrar o tronco até $1,80 \mathrm{~m}$ e o controle do mato com capinas nas entrelinhas.

O material genético estudado neste trabalho é constituído de 28 progênies de meio-irmãos provenientes de sementes de polinização aberta, obtidas de 28 clones parentais selecionadas fenotipicamente em uma população de $H$. brasiliensis, constituída de material de procedência asiática estabelecida em 1952, no Instituto Agronômico(IAC), em Campinas (GonçALves et al., 1991). Os materiais genéticos (28 matrizes) selecionados foram os seguintes: AVROS 49, AVROS 255, AVROS 352, AVROS 363, AVROS 1328, AVROS 1126, AVROS 1279, 
C 228, C 256, C 259, C 290, C 297, C 318, GT 127, GT 711, PB 49, PB 86, PB 5/63, PR 107, RRIM 501, RRIM 512, RRIM 513, RRIM 600, RRIM 623, RRIM 626, RRIM 1526, Tjir 1, Tjir 16.

As mudas foram produzidas a partir de sementes coletadas de clones estabelecidos no Pólo Noroeste Paulista, em Votuporanga (SP), pertencente à Agência Paulista de Tecnologia dos Agronegócios. As progênies foram instaladas sob o delineamento de blocos ao acaso com 28 tratamentos, três repetições e dez plantas por parcela, no espaçamento de $3 \mathrm{~m} \times 2$ $\mathrm{m}$ em linhas simples.

Aos três anos de idade, as progênies foram avaliadas quanto aos caracteres: a) altura total $(\mathrm{cm})$; b) diâmetro $(\mathrm{mm})$; c) Produção de borracha seca $(\mathrm{g})$ : obtida pelo teste Hamaker-Morris-Mann (HMM), modificado para plantas de três anos de idade (TAN e Subramanian, 1976), utilizando-se a média de produção de borracha seca de 30 cortes por plântula. O painel de sangria foi aberto a $20 \mathrm{~cm}$ do solo, pelo sistema $\mathrm{S} / 2 \mathrm{~d} / 3$, no total de 35 cortes, descartandose as cinco primeiras amostras que correspondem à fase de amansamento do painel. A nomenclatura S/2 corresponde ao corte em meia espiral, e a nomenclatura $d / 3$ expressa o intervalo entre sangrias, ou seja, uma sangria a cada três dias; d) Produtividade ( $\mathrm{g} \mathrm{cm}^{-2}$ ): obtida através da razão entre os valores de produção de borracha seca $(\mathrm{g})$ por $\mathrm{cm}^{2}$, provenientes dos valores de diâmetro das progênies (TAN e SUBRAMANIAN, 1976).

As variáveis foram analisadas usando-se a metodologia de modelo linear misto (univariado aditivo) adaptado por RESENDE (2002), consistindo da seguinte equação:

$$
\mathrm{y}=\mathrm{Xb}+\mathrm{Za}+\mathrm{Wc}+\mathrm{e}, \text { em que: }
$$

$y, b, a, c$ e e: vetores de dados, dos efeitos de blocos (aleatório), de efeitos genéticos aditivos (aleatório), de efeitos de parcela (aleatório) e de erros aleatórios, respectivamente.

$\mathrm{X}, \mathrm{Z}$ e W: matrizes de incidência para $\mathrm{b}$, a e c, respectivamente.

\section{Distribuições e estruturas de médias e variâncias}

$$
\begin{aligned}
& \mathrm{y} \mid \mathrm{b}, \mathrm{V} \sim \mathrm{N}(\mathrm{Xb}, \mathrm{V}) \\
& \mathrm{a} \mid \mathrm{A}, \hat{\sigma}_{\mathrm{A}}^{2} \sim \mathrm{N}\left(0, \mathrm{~A} \sigma_{\mathrm{a}}^{2}\right) \\
& \mathrm{d} \hat{\sigma}_{\mathrm{c}}^{2} \sim \mathrm{N}\left(0, \mathrm{I} \sigma_{\mathrm{c}}^{2}\right) \\
& \mathrm{e} \hat{\sigma}_{\mathrm{e}}^{2} \sim \mathrm{N}\left(0, \mathrm{I} \sigma_{\mathrm{e}}^{2}\right) \\
& \operatorname{Cov}\left(\mathrm{a}, \mathrm{c}^{\prime}\right)=0 ; \operatorname{Cov}\left(\mathrm{a}, \mathrm{e}^{\prime}\right)=0 ; \operatorname{Cov}\left(\mathrm{c}, \mathrm{e}^{\prime}\right)=0 \\
& \text { ou seja: }
\end{aligned}
$$

$$
\begin{aligned}
& \mathrm{E}\left[\begin{array}{l}
\mathrm{Y} \\
\mathrm{a} \\
\mathrm{C} \\
\mathrm{e}
\end{array}\right]=\left[\begin{array}{c}
\mathrm{Xb} \\
0 \\
0 \\
0
\end{array}\right] \text { e } \operatorname{Var}\left[\begin{array}{c}
\mathrm{Y} \\
\mathrm{a} \\
\mathrm{C} \\
\mathrm{e}
\end{array}\right]=\left[\begin{array}{cccc}
\mathrm{V} & \mathrm{ZG} & \mathrm{WC} & \mathrm{R} \\
\mathrm{GZ} & \mathrm{G} & 0 & 0 \\
\mathrm{CW} & 0 & \mathrm{C} & 0 \\
\mathrm{R} & 0 & 0 & \mathrm{R}
\end{array}\right] \text {, em que: } \\
& \mathrm{G}=\mathrm{A} \hat{\sigma}_{\mathrm{A}}^{2} \\
& \mathrm{R}=\mathrm{I} \hat{\sigma}_{\mathrm{C}}^{2} \\
& \mathrm{C}=\mathrm{I} \hat{\sigma}_{\mathrm{e}}^{2} \\
& \mathrm{~V}=\mathrm{ZA} \hat{\sigma}_{\mathrm{a}}^{2} \mathrm{Z}+\mathrm{W} \mathrm{I} \hat{\sigma}_{\mathrm{C}}^{2} \mathrm{~W}+\mathrm{I} \hat{\sigma}_{\mathrm{e}}^{2}=\mathrm{ZGZ}+\mathrm{W} \mathrm{CW}+\mathrm{R} .
\end{aligned}
$$

Equações de modelo misto

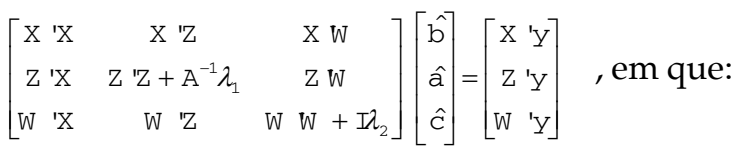

$$
\begin{aligned}
& \lambda_{1}=\frac{\hat{\sigma}_{\mathrm{e}}^{2}}{\hat{\sigma}_{\mathrm{a}}^{2}}=\frac{1-\hat{h}^{2}-\mathrm{c}^{2}}{\hat{h}^{2}} ; \quad \lambda_{2}=\frac{\hat{\sigma}_{\mathrm{e}}^{2}}{\hat{\sigma}_{\mathrm{a}}^{2}}=\frac{1-\hat{h}^{2}-\hat{\mathrm{c}}^{2}}{\hat{\mathrm{c}}^{2}} \\
& \hat{h}^{2}=\frac{\hat{\sigma}_{a}^{2}}{\hat{\sigma}_{a}^{2}+\hat{\sigma}_{c}^{2}+\hat{\sigma}_{e}^{2}}=\text { herdabilidade individual no }
\end{aligned}
$$

sentido restrito no bloco;

$$
\hat{h}_{\mathrm{mp}}^{2}=\frac{0,25 \hat{\sigma}_{\mathrm{a}}^{2}}{0,25 \hat{\sigma}_{\mathrm{a}}^{2}+\hat{\sigma}_{\mathrm{c}}^{2} / \mathrm{b}+\hat{\sigma}_{\mathrm{e}}^{2} /(\mathrm{nb})}=\text { herdabilidade média }
$$

de progênies no sentido restrito no bloco;

$\hat{c}^{2}=\hat{\sigma}_{c}^{2} /\left(\hat{\sigma}_{a}^{2}+\hat{\sigma}_{c}^{2}+\hat{\sigma}_{e}^{2}\right)=$ correlação devida ao ambiente comum da parcela;

$\hat{\sigma}_{a}^{2}=$ variância genética aditiva;

$\hat{\sigma}_{\mathrm{c}}^{2}=$ variância entre parcelas;

$\hat{\sigma}_{\mathrm{e}}^{2}=$ variância residual (ambiente dentro de parcelas + não aditiva);

$\mathrm{A}=$ matriz de correlação genética aditiva entre os indivíduos em avaliação.

As estimativas dos parâmetros genéticos foram obtidas pelo procedimento REML (restricted maximum likelihood), a partir de iterações nas equações de modelo misto.

\section{RESULTADOS E DISCUSSÃO}

Os resultados referentes às estimativas dos parâmetros genéticos para os caracteres altura, diâmetro, produção de borracha seca $(\mathrm{g})$ e produtividade $\left(\mathrm{g} \mathrm{cm}^{-2}\right)$ das plantas são apresentados na tabela 1.

Os coeficientes de variação genética individual $\left(\mathrm{CV}_{\mathrm{g}} \%\right)$, que expressam, em percentagem da média geral, a quantidade de variação genética existente, tiveram valores moderados para os caracteres altura, diâmetro, produção de borracha seca e produtividade $(15,06$; $19,42 ; 18,04$ e 27,61$)$ respectivamente. 
Tabela 1. Estimativas de parâmetros genéticos para os caracteres altura, diâmetro, produção de borracha seca e produtividade em progênies de seringueira, no município de Dois Irmãos do Buriti (MS)

\begin{tabular}{lcccc}
\hline Estimativas & Altura & Diâmetro & Produção de borracha seca & Produtividade \\
\hline & $\mathrm{cm}$ & $\mathrm{mm}$ & $\mathrm{g}$ & $\mathrm{g} \mathrm{cm}$ \\
$\left(\hat{h}_{\mathrm{a}}^{2}\right)$ & 0,328 & 0,240 & 0,089 & 0,510 \\
$\left(\hat{h}_{\text {mp }}^{2}\right)$ & 0,656 & 0,578 & 0,686 & 0,751 \\
$\left(\hat{\sigma}_{\mathrm{a}}^{2}\right)$ & 833,898 & 7,245 & 0,028 & 0,110 \\
$\left(\hat{\sigma}_{\text {parc }}^{2}\right)$ & 42,278 & 0,478 & 0,004 & 0,004 \\
$\left(\hat{\sigma}_{\mathrm{e}}^{2}\right)$ & 1665,579 & 22,500 & 0,284 & 0,105 \\
$\left(\hat{\sigma}_{\mathrm{f}}^{2}\right)$ & 2541,754 & 30,223 & 0,215 & 0,217 \\
\hline Média geral & 191,688 & 13,856 & 0,931 & 1,196 \\
$(\mathrm{CV}$ \% $)$ & 15,065 & 19,426 & 18,046 & 27,610 \\
$(\mathrm{CV}$ \% $)$ & 9,457 & 14,379 & 19,826 & 13,750 \\
\hline
\end{tabular}

Herdabilidade individual no sentido restrito no bloco $\left(\hat{h}_{a}^{2}\right)$; herdabilidade média de progênie $\left(\hat{h}_{\operatorname{mp}}^{2}\right)$; variância genética aditiva $\left(\hat{\sigma}_{\mathrm{a}}^{2}\right)$; variância ambiental entre parcelas $\left(\hat{\sigma}_{\text {parc }}^{2}\right)$; variância residual dentro de parcela (ambiental + não aditiva, $\left.\hat{\sigma}_{\mathrm{e}}^{2}\right)$; variância fenotípica individual $\left(\hat{\sigma}_{\mathrm{f}}^{2}\right)$; coeficiente de variação genética $\left(\mathrm{CV}_{\mathrm{g}} \%\right)$; coeficiente de variação ambiental dentro de parcelas $\left(\mathrm{CV}_{\mathrm{e}} \%\right)$.

Esses resultados são condizentes àqueles constados por MoReTI et al. (1994), Boock et al. (1995) e Costa et al. (2000a) para os três primeiros caracteres e revelam que a população pode ser considerada apropriada para o programa de melhoramento genético.

Os coeficientes de variação residual $\left(\mathrm{CV}_{\mathrm{e}} \%\right)$ tiveram valores de $9,45 \%$ para altura, $14,37 \%$ para diâmetro, 19,82\% para produção de borracha seca e $13,75 \%$ para produtividade, considerados de moderadas magnitudes por GARCIA et al. (1989).

As estimativas dos coeficientes de herdabilidades individuais $(0,33 ; 0,24$ e 0,51$)$ respectivamente, para os caracteres altura, diâmetro e produtividade foram considerados expressivos. Para o caráter produção de borracha seca a estimativa do coeficiente de herdabilidade individual, no sentido restrito $(9 \%)$, foi de baixa magnitude, porém há excelente possibilidade de seleção, pois conduziram à estimativa da herdabilidade, em nível de médias de família de $68 \%$. Portanto, as estimativas obtidas poderão maximizar os ganhos genéticos com seleção na sequência das avaliações. Os resultados são condizentes com aqueles verificados por PaIVA et al. (1982), Alika (1985) e Costa et al. (2000a) para a seringueira, excetuando-se produtividade, não estudados nos trabalhos citados.

Os valores expressivos das herdabilidades de médias de progênies para todos os caracteres estudados, indicam que a seleção pode ser mais efetiva utilizando-se as informações das famílias. Tais resultados são próximos aos observados na literatura relativa à seringueira para os caracteres altura, diâmetro e produção de borracha seca (GILBERT et al., 1973; NGa e Subramanian, 1974; Moreti et al., 1994, Costa et al., 2000b e GonçAlves et al., 2005).

$\mathrm{Na}$ tabela 2 são apresentados os valores fenotípicos, genéticos aditivos, ganhos genéticos preditos e nova média da população referente à recombinação dos melhores indivíduos oriundos de seis progênies para o caráter altura $(\mathrm{cm})$, no Município de Dois Irmãos do Buriti (MS).

Constatou-se que os indivíduos das progênies 21, 8, 2, 3 e 19 foram promissores quando comparados aos indivíduos das demais progênies. Os ganhos genéticos obtidos variaram de $38,0 \mathrm{~cm}$ a $49,9 \mathrm{~cm}$, elevando a nova média da população, após um ciclo de seleção, de 191,7 cm para 241,6 cm, considerando os cinco indivíduos mais bem classificados para o caráter altura. Esses resultados estimulam a seqüência das avaliações do programa de melhoramento genético, associado à produção de borracha seca.

A acurácia entre os valores genéticos preditos e os verdadeiros foram de 0,80 para altura, 0,76 para diâmetro, 0,62 para produção de borracha seca e 0,86 para produtividade (Tabelas 2, 3, 4 e 5). Segundo RESENDE (2002), a acurácia é uma medida que está associada à precisão na seleção, sendo o principal componente do progresso genético que pode ser alterado visando maximizar o ganho. A acurácia pode ser incrementada por meio de uma experimentação mais adequada, mantendo-se o mesmo tamanho do experimento, porém, alterandose o número de indivíduos por parcela e repetições (RESENDE 2002). 
Tabela 2. Valores fenotípicos, genéticos aditivos, ganhos genéticos preditos e nova média da população referente à recombinação dos melhores indivíduos (árvores) oriundos de seis progênies, para o caráter altura (cm) de seringueira, no Município de Dois Irmãos do Buriti, (MS)

\begin{tabular}{|c|c|c|c|c|c|}
\hline Progênies & Árvore & Valores fenotípicos & Valores genéticos $(u+a)^{*}$ & Ganho genético & Nova Média \\
\hline & & & & $\mathrm{cm}$ & \\
\hline $\begin{array}{l}21 \\
\text { (AVROS } 363 \text { x C 318) }\end{array}$ & 1 & 359,0 & 241,57 & 49,88 & 241,57 \\
\hline 8 (AVROS 363 x RRIM 501) & 2 & 325,0 & 239,67 & 48,93 & 240,62 \\
\hline $\begin{array}{l}2 \\
(\text { AVROS } 1279 \text { x PB 49) }\end{array}$ & 6 & 357,0 & 236,96 & 47,71 & 239,40 \\
\hline $\begin{array}{l}3 \\
(\mathrm{C} 228 \times \mathrm{PB} 5 / 63)\end{array}$ & 1 & 317,0 & 234,63 & 46,52 & 238,20 \\
\hline 8 (AVROS 363 x RRIM 501) & 2 & 301,0 & 232,50 & 45,38 & 237,06 \\
\hline $\begin{array}{l}19 \\
\text { (C } 228 \times \text { Tjir } 16)\end{array}$ & 2 & 303,0 & 231,44 & 44,44 & 236,13 \\
\hline $\begin{array}{l}3 \\
(\mathrm{C} 228 \times \mathrm{PB} 5 / 63)\end{array}$ & 1 & 281,0 & 229,20 & 43,45 & 235,14 \\
\hline $\begin{array}{l}5 \\
(\mathrm{C} 197 \times \mathrm{PB} 49)\end{array}$ & 4 & 311,0 & 224,00 & 42,18 & 233,87 \\
\hline $\begin{array}{l}19 \\
\text { (C } 228 \times \text { Tjir } 16)\end{array}$ & 2 & 277,0 & 224,96 & 41,19 & 232,88 \\
\hline $\begin{array}{l}3 \\
(\mathrm{C} 228 \times \text { ×B 5/63) }\end{array}$ & 1 & 261,0 & 223,12 & 40,21 & 231,90 \\
\hline Acurácia seletiva & - & - & - & - & 0,80 \\
\hline
\end{tabular}

${ }^{*} \mathrm{u}+\mathrm{a}=$ valor genético aditivo predito.

Na tabela 3 são apresentados os valores fenotípicos, genéticos aditivos, ganhos preditos e nova média da população das 10 melhores progênies para o caráter diâmetro $(\mathrm{mm})$ em seringueira, no Município de Dois Irmãos do Buriti (MS).

Observa-se que os indivíduos da progênie 20 foram predominantes entre os mais promissores, com valores expressivos e constituem os 10 melhores materiais genéticos classificados, para o caráter diâmetro. De forma similar ao caráter altura, avaliações posteriores em idades mais avançadas poderão confirmar o desempenho desses materiais para efeito de seleção, visando maximizar o ganho genético. SIMEÃo et al. (2000) enfatizaram que os valores genéticos preditos em relação a todos os indivíduos candidatos possibilitam estabelecer a melhor estratégia para o aumento da eficiência do melhoramento.

Na tabela 4 são apresentados os valores fenotípicos, genéticos aditivos ganhos preditos e nova média da população das melhores progênies para $o$ caráter produtividade de borracha seca (g) em seringueira, no Município de Dois Irmãos do Buriti (MS).

Observa-se que indivíduos das progênies 21, 5, 19 e 4 tiveram melhor desenvolvimento em termos de produção de borracha seca, com ganhos genéticos que variaram de 0,14 a 0,21 gramas, elevando a nova média da população para o caráter, após um ciclo de seleção, de 0,93 grama para 1,14 gramas. Esse fato denota o potencial da população para produção de borracha seca.

Os ganhos adicionais obtidos com a seleção são coerentes com a herdabilidade individual $(8,9 \%)$ e o coeficiente de variação genética $(18,04 \%)$ encontrados para o caráter está contido na tabela 1. Os referidos parâmetros, juntamente com os ganhos, sugerem boas possibilidades de progresso genético na seqüência das avaliações em idades mais avançadas.

Na tabela 5, são apresentados os valores fenotípicos, genéticos aditivos ganhos preditos e nova média da população dos 10 melhores indivíduos das seis melhores progênies para o caráter produtividade $\left(\mathrm{g} \mathrm{cm}^{-2}\right)$ em seringueira, no Município de Dois Irmãos do Buriti (MS).

Os resultados demonstraram superioridade dos indivíduos das progênies 5, 3 e 21 para o caráter produção de borracha seca, de forma análoga aos valores encontrados para os caracteres altura e produção de borracha seca. Ressalta-se os valores elevados de ganho genético variando de 0,55 a 0,88. Tais resultados refletem de forma direta, os parâmetros genéticos herdabilidades individual $(0,51)$ e média de progênies $(0,75)$ (tabela 1$)$. 
Tabela 3. Valores fenotípicos, genéticos aditivos, ganhos genéticos preditos e nova média da população, das 10 melhores progênies para o caráter diâmetro $(\mathrm{mm})$ de seringueira, no Município de Dois Irmãos do Buriti (MS)

\begin{tabular}{|c|c|c|c|c|c|}
\hline Progênies & Árvore & Valores fenotípicos & Valores genéticos $(u+a)^{*}$ & Ganho genético & Nova Média \\
\hline & & & & $\mathrm{mm}$ & \\
\hline $\begin{array}{l}20 \\
\text { (AVROS } 255 \text { x RRIM 600) }\end{array}$ & 2 & 85,0 & 29,87 & 16,02 & 29,87 \\
\hline $\begin{array}{l}20 \\
\text { (AVROS } 255 \text { x RRIM 600) }\end{array}$ & 1 & 23,0 & 17,81 & 9,99 & 23,84 \\
\hline $\begin{array}{l}20 \\
\text { (AVROS } 255 \text { x RRIM 600) }\end{array}$ & 2 & 22,0 & 17,50 & 7,87 & 21,73 \\
\hline $\begin{array}{l}20 \\
\text { (AVROS } 255 \text { x RRIM 600) }\end{array}$ & 3 & 21,0 & 17,42 & 6,80 & 20,65 \\
\hline $\begin{array}{l}20 \\
\text { (AVROS } 255 \text { x RRIM 600) }\end{array}$ & 3 & 21,0 & 17,31 & 6,13 & 19,98 \\
\hline & 1 & 20,0 & 17,11 & 5,65 & 19,50 \\
\hline $\begin{array}{l}20 \\
\text { (AVROS } 255 \text { x RRIM 600) }\end{array}$ & 4 & 19,0 & 16,92 & 5,28 & 19,13 \\
\hline $\begin{array}{l}20 \\
\text { (AVROS } 255 \text { x RRIM 600) }\end{array}$ & 4 & 18,0 & 16,84 & 5,00 & 18,85 \\
\hline $\begin{array}{l}20 \\
\text { (AVROS } 255 \times \text { RRIM 600) }\end{array}$ & 5 & 17,0 & 16,65 & 4,75 & 18,60 \\
\hline $\begin{array}{l}20 \\
\text { (AVROS } 255 \text { x RRIM 600) }\end{array}$ & 5 & 16,0 & 16,34 & 4,52 & 18,38 \\
\hline Acurácia seletiva & & & & & 0,76 \\
\hline
\end{tabular}

${ }^{*} \mathrm{u}+\mathrm{a}=$ valor genético aditivo predito.

Tabela 4. Valores fenotípicos, genéticos aditivos, ganhos genéticos preditos e nova média da população das melhores progênies para o caráter produção de borracha seca (g) de seringueira, no Município de Dois Irmãos do Buriti (MS)

\begin{tabular}{|c|c|c|c|c|c|}
\hline Progênies & Árvore & Valores fenotípicos & Valores genéticos $(\mathrm{u}+\mathrm{a})^{*}$ & Ganho genético & Nova Média \\
\hline & & & & g & \\
\hline $\begin{array}{l}21 \\
(\text { AVROS } 363 \text { x C 318) }\end{array}$ & 4 & 3,40 & 1,14 & 0,21 & 1,14 \\
\hline $\begin{array}{l}5 \\
(\mathrm{C} 197 \times \text { × } 1949)\end{array}$ & 1 & 3,40 & 1,13 & 0,20 & 1,13 \\
\hline $\begin{array}{l}5 \\
(\mathrm{C} 197 \times \text { ×B 49) }\end{array}$ & 1 & 2,60 & 1,08 & 0,18 & 1,12 \\
\hline (AVROS 1328 x PB 86) & 4 & 2,33 & 1,08 & 0,17 & 1,11 \\
\hline$(\mathrm{C} 228 \times$ Tjir 16$)$ & 4 & 2,03 & 1,07 & 0,17 & 1,10 \\
\hline (AVROS $1328 \times$ PB 86) & 5 & 2,33 & 1,07 & 0,16 & 1,09 \\
\hline $\begin{array}{l}19 \\
\text { (C } 228 \times \text { Tjir } 16)\end{array}$ & 1 & 2,17 & 1,07 & 0,16 & 1,09 \\
\hline $\begin{array}{l}3 \\
(\mathrm{C} 228 \times \mathrm{PB} 5 / 63)\end{array}$ & 5 & 3,30 & 1,07 & 0,16 & 1,09 \\
\hline $\begin{array}{l}4 \\
\text { (AVROS } 1328 \times \text { PB 86) }\end{array}$ & 1 & 2,20 & 1,06 & 0,15 & 1,09 \\
\hline$(\mathrm{C} 228 \times$ Tjir 16$)$ & 2 & 1,90 & 1,05 & 0,15 & 1,08 \\
\hline Acurácia seletiva & & & & & 0,62 \\
\hline
\end{tabular}

* $\mathrm{u}+\mathrm{a}=$ valor genético aditivo predito. 
Tabela 5. Valores fenotípicos, genéticos aditivos, ganhos genéticos preditos e nova média da população dos 10 melhores indivíduos das seis melhores progênies para o caráter produtividade $\left(\mathrm{g} \mathrm{cm}^{-2}\right)$ de seringueira, no Município de Dois Irmãos do Buriti (MS)

\begin{tabular}{|c|c|c|c|c|c|}
\hline Progênies & Árvore & Valores fenotípicos & Valores genéticos $(u+a)^{*}$ & Ganho genético & Nova Média \\
\hline & & & & $\mathrm{g} \mathrm{m}^{-2}$ & \\
\hline $\begin{array}{l}5 \\
(\text { C } 197 \times \text { ×B 49) }\end{array}$ & 1 & 3,0 & 2,078 & 0,882 & 2,078 \\
\hline $\begin{array}{l}3 \\
(\mathrm{C} 228 \times \mathrm{PB} 5 / 63)\end{array}$ & 5 & 3,0 & 2,077 & 0,882 & 2,077 \\
\hline $\begin{array}{l}21 \\
(\text { AVROS } 363 \times \text { C } 318)\end{array}$ & 4 & 3,0 & 2,054 & 0,874 & 2,069 \\
\hline $\begin{array}{l}5 \\
(\mathrm{C} 197 \text { x PB 49) }\end{array}$ & 1 & 2,0 & 1,652 & 0,769 & 1,965 \\
\hline (C 228 x PB 5/63) & 4 & 2,0 & 1,638 & 0,704 & 1,890 \\
\hline (AVROS $1328 \times$ PB 86) & 1 & 2,0 & 1,603 & 0,655 & 1,850 \\
\hline (AVROS 1328 x PB 86) & 5 & 2,0 & 1,603 & 0,619 & 1,815 \\
\hline $\begin{array}{l}4 \\
(\text { AVROS } 1328 \times \text { PB 86) }\end{array}$ & 4 & 2,0 & 1,598 & 0,592 & 1,788 \\
\hline $\begin{array}{l}7 \\
\text { (C } 297 \text { x PR 107) }\end{array}$ & 2 & 2,0 & 1,589 & 0,570 & 1,766 \\
\hline $\begin{array}{l}1 \\
\text { (C } 256 \times \text { RRIM 600) }\end{array}$ & 5 & 2,0 & 1,577 & 0,551 & 1,747 \\
\hline Acurácia seletiva & & & & & 0,86 \\
\hline
\end{tabular}

${ }^{*} \mathrm{u}+\mathrm{a}=$ valor genético aditivo predito.

Por esses resultados demonstra-se a importância da variância aditiva no controle do caráter produção de borracha seca, concordando com os relatos de Simmonds (1989) e TAN e Subramanian (1976). Além disso, denotam que a heterose pode ser efetivamente explorada nas condições da população em estudo.

\section{CONCLUSÕES}

1. As estimativas de herdabilidades individuais associadas à média de progênie sugerem a maximização dos ganhos genéticos com a seleção na população.

2. A seleção com inclusão do caráter produtividade é promissora e deve ser utilizada na sequência das avaliações, subsidiando o programa de melhoramento genético da espécie no estado de Mato Grosso do Sul, especialmente no Bioma Cerrado.

\section{REFERÊNCIAS}

ALIKA, J.E. Heritability and genotypic gain from selection rubber (Hevea brasiliensis). Silvae Genetica, v.34, p.1-4, 1985.
BOOCK, M.V.; GONÇALVES, P.S.; BORTOLETTO, N.; MARTINS, A.L.M. Herdabilidade, variabilidade genética e ganhos genéticos para produção e caracteres morfológicos em progênies jovens de seringueira. Pesquisa Agropecuária Brasileira, v.30, p.673-681, 1995.

COSTA, R.B.; RESENDE, M.D.V.; ARAUJO, A.J.; GONÇALVES, P. de S.; BOTOLETTO, N. Seleção combinada univariada e multivariada aplicada ao melhoramento genético da seringueira. Pesquisa Agropecuária Brasileira, v.35, p.381-388, 2000a.

COSTA, R.B.; RESENDE, M.D.V.; GONÇALVES, P.S. Selection and genetic gain in populations of Hevea brasiliensis with a mixed mating system. Genetics and Molecular Biology, v.23, p.671-679, 2000b.

COSTA, R.B.; RESENDE, M. D. V.; CONTINI, A. Z.; REGO, F. L. H.; ROA, R. A. R.; MARTINS, W. J. Avaliação genética dentro de progênies de erva-mate (Ilex paraguariensis St. Hil.), na região de Caarapó, MS, pelo procedimento REML/BLUP. Ciência Florestal, v.15, p.371-376, 2005.

GARCIA, C.H. Tabela para classificação do coeficiente de variação. Piracicaba: Instituto de Pesquisas e Estudos Florestais. IPEF, 1989. 12p. (Circular Técnica 171)

GILBERT, N.E.; DODDS, K.S.; SUBRAMANIAN, S. Progress of breeding investigations with Hevea brasiliensis. Analysis of data from earlier crosses. Journal of the Rubber Research Institute of Malaysia, v.3, p.365-380, 1973. 
GONÇALVES, P. de S.; CARDOSO, M.; BOAVENTURA, M.M.; COLOMBO, C.A.; ORTOLANI, A.A. Clones de Hevea: influência dos fatores ambientais na produção e recomendação para o plantio. Campinas, Instituto Agronômico 1991. 32p. (Boletim Técnico, 138)

GONÇALVES, P. de S.; MORAES, M.L.T.; BOROLETTO, N.; COSTA, R.B.; GONÇALVES, E.C.P. Genetic variation in growth traits and yield of rubber trees (Hevea brasiliensis) growing in the Brazilian state of São Paulo. Genetics and Molecular Biology, v.28, p.765-772, 2005.

MISSIO, R. F.; SILVA, A. M.; DIAS, L. A. S.; MORAES, M. L. T.; RESENDE, M. D. V. Estimates of genetic parameters and prediction of additive genetic values in Pinus kesya progenies. Crop breeding and applied biotechnology, v.5, p. 394-401, 2005.

MORETI, D.; GONÇALVES, P. de S.; GORGULHO, E.P.; MARTINS, A.L.M.; BORTOLETTO, N. Estimativas de parâmetros genéticos e ganhos esperados com a seleção de caracteres juvenis em progênies de seringueira. Pesquisa Agropecuária Brasileira, v.29, p.1099-1109, 1994.

NGA, B.H.; SUBRAMANIAN, S. Variation in Hevea brasiliensis. I. Yield and girth data of the 1937 hand pollinated seedlings. Journal of the Rubber Research Institute of Malaysia, v.24, p.69-74, 1974.

PAIVA, J.R.; MIRANDA FILHO, J.B.; SIQUEIRA, E.R.; VALOIS, A.C.C. Predição do ganho de alguns caracteres em seringueira em três esquemas de seleção. Pesquisa Agropecuária Brasileira, v.17, p.1646-1653, 1982.

PEREIRA, J.P. Seringueira: formação de mudas, manejo e perspectivas no noroeste do Paraná. Londrina: IAPAR, 1992. 60p. (IAPAR. Circular Técnica, 700)

RAIJ, B. V.; CANTARELLA, H.; QUAGGIO, J.A.; FURLANI, A.M.C. (Ed.). Recomendações de adubação e calagem para o Estado de São Paulo. 2.ed. Campinas: Instituto Agronômico/ Fundação IAC, 1997. 285p. (Boletim Técnico, 100)

RESENDE, M.D.V.; FERNANDES, J.S.C. Procedimento BLUP (Melhor Predição Linear Não Viciada) individual para delineamentos experimentais aplicados ao melhoramento florestal. Revista Matemática Estatística, v.17, p.89-107, 1999.

RESENDE, M.D.V. Genética biométrica e estatística no melhoramento de plantas perenes. Brasília: Embrapa Informação Tecnológica, 2002. 975p.

SIMEÃO, R.M.; STURION, J.A.; RESENDE, M.D.V. Avaliação Genética em erva-mate pelo procedimento BLUP individual multivariado sob interação genótipo $x$ ambiente. Pesquisa Agropecuária Brasileira, v.37, p.1589-1596, 2000.

SIMMONDS, N.W. Rubber breeding. In: WEBSTER, C.C.; BAULKWILL, W,J. (Eds.). Rubber. London: Longman, 1989. p.85-124.

TAN, H.; SUBRAMANIAN, S.A. A five-parent diallel cross analysis for certain characters of young Hevea seedlings. In: INTERNACIONAL RUBBER CONFERENCE, Kuala Lumpur 1975. Proceedings... v.2, p.13-16, 1976. 